\title{
ESTABLECIMIENTO DE UN PROGRAMA DE CIRUGÍA ROBÓTICA EN UN HOSPITAL.
}

\author{
Antonio Allona Almagro y Arturo Platas Sancho.
}

Servicio de Urología. Hospital Ruber Internacional. Madrid., España.

\begin{abstract}
Resumen.- La cirugía robótica se va abriendo paso poco a poco en la urología. Por el momento son pocos los centros que se han incorporado a esta nueva tecnología en España pero es previsible que este número aumente exponencialmente en los próximos años. Describimos la experiencia de nuestro centro para el establecimiento y desarrollo de un programa de cirugía robótica que lleva en funcionamiento desde julio de 2006. Es importante conocer alguna serie de premisas básicas que se deben cumplir para alcanzar unos resultados óptimos.
\end{abstract}

Palabras clave: Cirugía robótica. Programa. Establecimiento.

Summary.- Robotic surgery is expanding little by little in urology. At present only few centers have incorporated this new technology in Spain but it is predictable that this number will exponentially increase in a near future. We described the experience of our center establishing and developing a program of robotic surgery that it is operating since July 2006. It is important to know some basic premises that it is compulsory to fulfill to reach optimal results.

Keywords: Robotic surgery. Program. Center.

\section{INTRODUCCIÓN}

Las ventajas de la cirugía mínimamente invasiva son ampliamente aceptadas por la mayoría de los urólogos. Corta estancia hospitalaria, menor dolor en el postoperatorio, menor íleo paralítico y más rápida reincorporación a su actividad habitual son beneficios claramente en relación con la cirugía laparoscópica $(1,2)$. Sin embargo, la visión bidimensional del campo operatorio así como una limitación en la libertad de movimientos suponen un reto para el cirujano e indudablemente un riesgo para el paciente. La cirugía laparoscópica es, sin duda, la técnica ideal para procedimientos básicos que requieren de una mínima reconstrucción. Sólo un pequeño grupo de urólogos ha desarrollado una laparoscopia avanzada que les permite manipulaciones complejas como suturas, nudos, y por tanto una avanzada cirugía reconstructiva. Además el tiempo requerido para desarrollar esta capacidad es alto y por tanto no al alcance de la mayoría de los cirujanos (3).

Antes de establecer un programa de robótica en cualquier centro deben ser evaluados una serie de puntos fundamentales:

1. Especialidades quirúrgicas implicadas y procedimientos a realizar.

2. Desarrollo económico y marketing.

3. Quirófano y personal.

4. Programas de formación.

5. Control de resultados. 


\section{PROGRAMA DE CIRUgÍA ROBÓtICA MULTI- DISCIPLINAR}

El robot da Vinci® (Intuitive Surgical, Sunnyvale, California) permite al laparoscopista realizar unos procedimientos laparoscópicos avanzados de una forma más sencilla. Dos videocámaras acopladas permiten una visión tridimensional del campo operatorio con una magnificación ajustable de hasta 10X. Los instrumentos articulados del robot se mueven con los mismos 7 grados de libertad que las manos del cirujano en cirugía abierta traspasando a la parte distal de los instrumentos la capacidad que tiene la muñeca humana y mejorando el grado de giro de ésta. Por último, la eliminación del temblor conlleva una mayor precisión quirúrgica. Los movimientos además son ajustados por transmitirse en escala (por ejm: $3 \mathrm{~cm}$ de campo pasan a ser $1 \mathrm{~cm}$ en el extremo del instrumento).

El sistema da Vinci® consta de tres componentes mayores: la consola, la torre laparoscópica y la mesa quirúrgica. La consola constituye la interfase entre el cirujano y el robot. El cirujano ve dos videoimágenes que convergen en el visor permitiendo la visión como la natural que tenemos con los dos ojos, controla los instrumentos con controles manuales y maneja la cámara y los sistemas de corte y coagulación usando unos pedales con ambos pies. La configuración del sistema permite al primer cirujano tener el control directo de tres instrumentos (o de 2, según el robot tenga 4 ó 3 brazos) y de la cámara, mientras que en laparoscopia convencional solo controla un máximo de dos elementos. El sistema traslada los movimientos naturales de las manos del cirujano en precisos movimientos laparoscópicos dentro del paciente. La torre dispone de un monitor, que permite ver la operación a los cirujanos ayudantes, la fuente de luz de las cámaras, el insuflador, el grabador de vídeo y los controles de la cámara.

El robot da Vinci®, por todo lo antedicho, permite la realización de avanzadas técnicas laparoscópicas a cirujanos que no tienen un amplio entrenamiento en procedimientos videoendoscópicos y además mejora la calidad y aumento el número de procedimientos quirúrgicos distintos que pueden ser realizados con la laparoscopia convencional (4).

Los procedimientos quirúrgicos que pueden realizarse mediante cirugía robótica son los siguientes:
A. Cirugía cardiaca y torácica
- Reparación de la válvula mitral
- Extracción de la arteria mamaria interna
- Ventana pericárdica

- Esofagectomía

- Timectomía

- Derivación coronaria totalmente endoscópica (TE-

$\mathrm{CAB}$ ) sobre corazón funcionante y sobre corazón en parada

\section{B. Cirugía digestiva}

- Colecistectomía

- Funduplicatura de Nissen

- Anastomosis intestinal

- Resección de colon y de recto

- Gastroplastia

- Derivación gástrica

- Esplenectomía

C. Urología

- Prostatectomía radical (la cirugía estrella)

- Suprarrenalectomía

- Nefrectomía simple, radical y parcial

- Pieloplastia

- Cistectomía

- Cirugía ureteral y piélica de las litiasis

- Ureterectomía, reimplante ureterovesical, cirugía vesical parcial

- Linfadenectomía retroperitoneal

- Cirugía de la infertilidad: vaso-vasostomía y túbulovasostomía

D. Ginecología

- Histerectomía

- Reparación del suelo pélvico

- Miomectomías

- Cirugía tubárica

- Ooferectomía o similares

E. Otras especialidades

ORL: cirugía tiroidea

Cirugía vascular

Cirugía plástica

Neumocirugía

Idealmente varias especialidades quirúrgicas deben participar en el desarrollo de un programa de cirugía robótica, debido a la importante inversión inicial en la adquisición del equipo y a los altos costes directos e indirectos que conlleva el proyecto. Por este motivo muchos hospitales no pueden o no deciden implantar este tipo de programas (5).

\section{DESARROLLO ECONÓMICO Y MARKETING}

El desarrollo de un programa de cirugía robótica en un hospital público universitario constituye un hecho innovador y hay que considerar que afecta también a la formación de los médicos residentes y tiene posibles aplicaciones en el campo de la investigación. Es necesario investigar de forma continuada para demostrar la afirmación de que la robótica mejora la evolución clínica en ciertas intervenciones urológicas y de que ofrece claras ventajas sobre la 
cirugía laparoscópica convencional, de menor coste económico ésta. Otra posible ventaja es la de realización programas de formación dirigidos a profesionales que se inicien en la robótica, posibilidad también compartida por los hospitales privados, siendo fundamental la colaboración de la industria farmaceútica para su desarrollo.

Un aspecto importante para el centro hospitalario es conseguir el desarrollo de programas de formación y poder ofrecer servicios de telesupervisión quirúrgica en congruencia con los objetivos educativos del propio centro. La posibilidad de investigación, incluyendo la telecirugía, la aplicación de modalidades múltiples de imagen y el diseño de nuevos procedimientos también, puede ser una razón suficiente para establecer uno de estos programas.

El establecimiento de la cirugía robótica en un hospital privado tiene sus peculiaridades, principalmente en el terreno económico. El marketing es un elemento fundamental dado que la mayoría de las compañías de seguros no lo incluyen entre las prestaciones a sus asegurados. Sólo un progresivo aumento de enfermos tratados creará la justificación para que dichas empresas asuman este gasto. El coste inicial de la inversión es de casi 2.000.000 de euros en nuestro país, por lo que debe plantearse que su amortización no se realice a corto plazo. Es importante la implicación de posibles benefactores e involucrar a la mayoría de los servicios quirúrgicos del hospital que puedan hacer uso del robot. El contrato de mantenimiento anual de 120.000 euros y el costo de los recambios de los accesorios del robot, suponen un gasto estimado por paciente de 2.000 euros deben ser considerados. El desembolso final que puede realizar un paciente promedio sometido a prostatectomía radical robótica (todos los gastos incluidos), con una estancia media de tres días puede ascender a una cifra entre 15.000 y 30.000 euros según el número de pacientes por año y el centro hospitalario. La publicitación mediante anuncios publicitarios en publicaciones y otros medios de comunicación, mediante páginas web, presentaciones públicas en radio y televisión y la notificación a otros médicos que puedan remitir a los pacientes es indispensable. Sin embargo, no es comprensible que exista una actitud reacia por parte de especialistas que no conozcan o no puedan disponer de cirugía robótica, debiendo considerarse la realización de programas de formación dirigidos a estos médicos.

\section{QUIRÓFANO Y PERSONAL}

El quirófano ideal sería aquel que tuviera dedicado su uso exclusivo al sistema robótico y a todos sus elementos, para así evitar desplazamientos y los posibles riesgos de éstos sobre los componentes mecánicos y cables. Al menos se necesitan $52 \mathrm{~m} 2$, para permitir una adecuada disposición de las tres partes del robot, del carro de anestesia, de la mesa de quirófano, y una circulación idónea del personal quirúrgico. Se están ideando sistemas de proyección tridimensional que faciliten a los cirujanos ayudantes y enfermería seguir mejor el desarrollo de la cirugía laparoscópica (4).

En una cirugía robótica al menos son necesarios dos cirujanos, una enfermera ayudante, una enfermera o auxiliar de enfermera circulante y un anestesista.

El uso del robot da Vinci® con un cuarto brazo constituye un avance importante, pero no sustituye la labor del un cirujano ayudante, especialmente en la prostatectomía radical.

Es importante contar durante al menos las diez primera cirugías, con un tutor quirúrgico con experiencia y con un técnico de la compañía que nos ha suministrado el robot, con el fin de aclarar y dar solución a posibles problemas que puedan surgir durante la cirugía (6).

\section{FORMACIÓN DEL PERSONAL}

Mientras que la curva de aprendizaje asociada a la laparoscopia standard está claramente definida, las descripciones sobre el aprendizaje en relación al robot quirúrgico son muy escasas. Prasad compara los aprendizajes usando el robot Zeus y la laparoscopia convencional demostrando ser más rápido y sencillo el del robot. Otros trabajos muestran resultados similares para diferentes especialidades quirúrgicas y procedimientos específicos, poniendo de manifiesto que la robótica reduce la curva de aprendizaje de la cirugía laparoscópica $(7,8)$.

Actualmente el abordaje de la cirugía robótica como cirugía mínimamente invasiva es laparoscópico, pero utilizando movimientos "naturales" o propios de la cirugía abierta. Así se da una paradoja, ya que el cirujano abierto se encontrará cómodo con los movimientos, pero al tener la visión laparoscópica se sentirá incómodo, y por otro lado, el laparoscopista se sentirá cómodo con la visión del campo quirúrgico, pero deberá acostumbrarse a los movimientos "naturales" del robot que le son poco conocidos. En ambos casos se requerirá formación y práctica, aunque dicho aprendizaje es más corto que el necesario para la laparoscopia, y aquí radica otra de sus grandes ventajas como ya antes se ha comentado. Al ser el abordaje laparoscópico, es necesario tener unos mínimos cono- 
cimientos y práctica con las técnicas laparoscópicas, por lo menos una parte del equipo quirúrgico. Un cirujano "abierto" podrá operar con el robot, pero deberá contar con personas en su equipo con experiencia en laparoscopia. En cualquier caso, el ayudante en la mesa de operaciones es una pieza clave.

Hoy en día el programa de formación se basa principalmente en:

1. Selección del equipo adecuado.

2. Disponer ya de un robot para empezar a trabajar. 3. Un programa de formación que incluya un aprendizaje completo sobre el robot, sus componentes, características, y forma de trabajar, además de unas sesiones de entrenamiento, normalmente con animales o con cadáveres, para familiarizarse con la visión, los movimientos y el uso del robot. Se han desarrollado cursos intensivos multidisciplinarios de aprendizaje dirigidos a cirujanos cardiácos, ginecólogos, cirujanos generales, otorrinolaringólogos, cirujanos plásticos, neurocirujanos y urólogos, así como cursos monográficos dirigidos a cada especialidad. El curso de formación suele tener una duración de dos días.

4. Es interesante disponer de casos seleccionados previamente para empezar a trabajar con el robot en cuanto se haya realizado el curso de formación.

5. Hay que tener en cuenta que el programa de formación pretende solamente enseñar el robot y su uso, no la técnica quirúrgica ni la cirugía a realizar. Por este motivo, al principio, se deberían seleccionar casos de baja complejidad.

6. Durante las primeras intervenciones es fundamental contar con el soporte técnico necesario para el manejo y colocación del robot, principalmente de trócares, etc...

7. El programa debería completarse con sesiones periódicas "in vitro" para familiarizarse con los movimientos de los controles en operaciones como suturar, anudar, etc.. Para ello el sistema incluye una serie de instrumentos especiales de entrenamiento (9).

\section{CONTROL DE RESULTADOS}

Un indicador fundamental para evaluar el programa de cirugía robótica es comparar el volumen de cirugías realizadas en comparación con las existentes antes de su implantación.

Asimismo es importante evaluar diferentes parámetros clínicos, a intervalos variables, como tiempo quirúrgicos, estancia hospitalaria, pérdidas sanguíneas, dolor postoperatorio, tiempo hasta la reincorporación a la actividad normal, continencia, disfunción eréctil, márgenes quirúrgicos en la cirugía oncológica, etc.
Otros parámetros adicionales para evaluar calidad pueden ser el grado de satisfacción del consumidor y la comparación de los tiempos respecto a otros centros de referencia. También es necesaria la evaluación del rendimiento de estos sistemas a lo largo del tiempo, en comparación con los datos de referencia relativos a la morbilidad, la duración media de la hospitalización, los costes económicos globales, el coste económico por cada procedimiento (eficacia) y sobre todo el estado de los márgenes quirúrgicos en el caso de la cirugía oncológica. Por último debe introducirse instrumentos de medida para la determinación de la calidad de vida global de los pacientes tratados.

El estudio del rendimiento a lo largo del tiempo en comparación con los parámetros de valoración de la calidad internos va a determinar si la combinación de la cirugía robótica con las modalidades emergentes de bioimagen médica va a compensar la falta de información háptica. En los estudios que se realicen en el futuro, se determinarán y analizarán los resultados para comprobar esta hipótesis.

\section{BIBLIOGRAFÍA y LECTURAS RECOMENDADAS ( ${ }^{*}$ lectura de interés $y^{* *}$ lectura fundamental)}

*1. JATZKO, G.R.; LISBORG, P.H.; PERTL, A.M. y cols.: "Multivariate comparison of complications after laparoscopic cholecystectomy". Ann. Surg., 221: 381, 1995.

*2. GLASER, F.; SANNWALD, G.A.; BURH, H.J.: “General stress response to conventional and laparoscopic cholecystectomy”. Ann. Surg., 221: 372, 1995.

**3. BALLANTYNE, G.H.: "The pitfalls of laparoscopic surgery: challenges for robotics and telerobotic surgery". Surg. Laparosc. Endosc. Percutan. Tech., 12: 1, 2002.

**4. STEERS, W.D.; LEBEAU, S.; CARDELLA, J. Y cols.: "Establecimiento de un programa de cirugía robótica". Clín Urol North, 4: 729.

*5. HANLY, E.J.; MAROHN, M.R.; BACHMAN, S.L. y cols.: "Multiservice laparoscopic surgical training using daVinci surgical system”. Am. J. Surg, 187: 309, 2004.

**6. CHITWOOD, W.R.; NIFONG, L.W.; CHAPMAN, W.H. y cols.: "Robotic surgical training in an academic institution". Ann. Surg., 234, 4: 475, 2001.

*7. PRASSAD, S.M.; MANIAR, H.S.; SOPER, N.J. "The effect of robotic assistance on learning curves for basic laparoscopic skills”. Am. J. Surg. , 183: 702, 2002.

*8. RODRIGUES NETTO, N. JR.; MITRE, A.I.; LIMA, S.V. y cols.: "Telementoring between Brazil and the United Status: Initial experience". J. Endourol., 17; 4:217, 2003

*9. BALLANTYNE, G.H.; KELLEY, W.E.: "Granting clinical privileges for telerobotic surgery". Surg Laparosc Endosc Percutan Tech. 12; 1:17. 2002. 